\title{
Star Formation in Disk Galaxies
}

\author{
Rosemary F. G. Wyse ${ }^{1}$, Annette M. N. Ferguson ${ }^{1}$, \\ Jay S. Gallagher ${ }^{2}$ and Deidre A. Hunter ${ }^{3}$ \\ ${ }^{1}$ Department of Physics and Astronomy, The Johns Hopkins University, \\ Baltimore, MD 21218, USA \\ wyse@pha.jhu.edu \\ ${ }^{2}$ Department of Astronomy, University of Wisconsin-Madison, Madison, WI 53706, USA \\ ${ }^{3}$ Lowell Observatory, Flagstaff, AZ 86001, USA
}

Received 1997 August 15, accepted 1997 December 1

\begin{abstract}
We present results, some preliminary, from a major new study of the star formation properties of a sample of nearby disk galaxies (Ferguson 1997). Our emphasis is on the faint outer regions of disks. $\mathrm{H} \alpha$ images, combined with broad-band images and spectroscopy of HII regions, constrain the present and past star formation rates and chemical enrichment. These data also allow study of faint diffuse ionised gas, which traces the influence of massive stars on their environment, and the structure of the interstellar medium.
\end{abstract}

Keywords: galaxies: ISM — galaxies: evolution — galaxies: abundances — stars: formation

\section{Introduction}

Determination of the physics behind the process of star formation, in addition to being of importance in its own right, is an integral part of the larger question of galaxy formation and evolution. Indeed, given that we use luminous galaxies as tracers of large-scale structure, even our understanding of cosmology ultimately depends on an understanding of what causes stars to form. Normal late-type disk galaxies are the sites of a significant fraction of the star formation in the local Universe, and understanding the rate of star formation in these systems is of obvious significance. The effects of star formation on the interstellar medium (ISM) are also highly relevant to understanding galaxy formation and evolution.

The current paradigm for galaxy formation and evolution invokes hierarchical clustering, in that gravitational collapse occurs first on small scales, and subsequently these systems merge to form galaxies, with baryons being only around $10 \%$ of the total gravitating matter (e.g. Silk \& Wyse 1993). The merging history of 'galaxies' and the rate at which the baryons turn into stars must play some role in the origins of the Hubble Sequence. In particular, feedback from massive stars may well determine the low efficiency of star formation in disks compared to that inferred for elliptical galaxies, and the difference in angular momentum distributions of disks and of ellipticals. The angular momentum of galaxies is believed to be generated by tidal torques between density perturbations prior to gravitational collapse, and is essentially independent of overdensity, scale, environment etc. Thus one is required to explain the origins of both rotationally-supported disk galaxies and 'pressure'-supported elliptical galaxies (the 'pressure' is random stellar motions) from the same initial values of the angular momentum parameter. This requires significant angular momentum rearrangement in proto-ellipticals, while the angular momentum distribution is conserved in proto-disks (e.g. Zurek, Quinn \& Salmon 1988). High-density stellar substructure will transport angular momentum most efficiently as it merges, via dynamical friction, whereas low-density, gaseous substructure may not. The latter then could form a disk galaxy. Hydrodynamic simulations of galaxy formation without star formation and feedback, but with gaseous cooling and viscosity, cannot form extended disks (e.g. Navarro \& Benz 1991). Attempts to include feedback from massive stars in such simulations are as yet incomplete, but it remains the most plausible solution to the problem of excessive concentration of proto-disks (e.g. Navarro \& Steinmetz 1997). Feedback from massive stars in the rather fragile potential of a disk will also keep the star formation efficiency low, allowing star formation to last a Hubble time.

We discuss here a program to address these questions from an observational study of a sample of nearby late-type disk galaxies. The spatial variation of present-day/recent massive-star formation is traced by $\mathrm{H} \alpha$ emission, while the past star formation is constrained by broad-band photometry, sensitive to older stars, and by spectroscopy of HII regions, to determine their chemical abundances. Special effort was made to study the outer regions of these galaxies, given that many of the 'laws' of disk-galaxy 


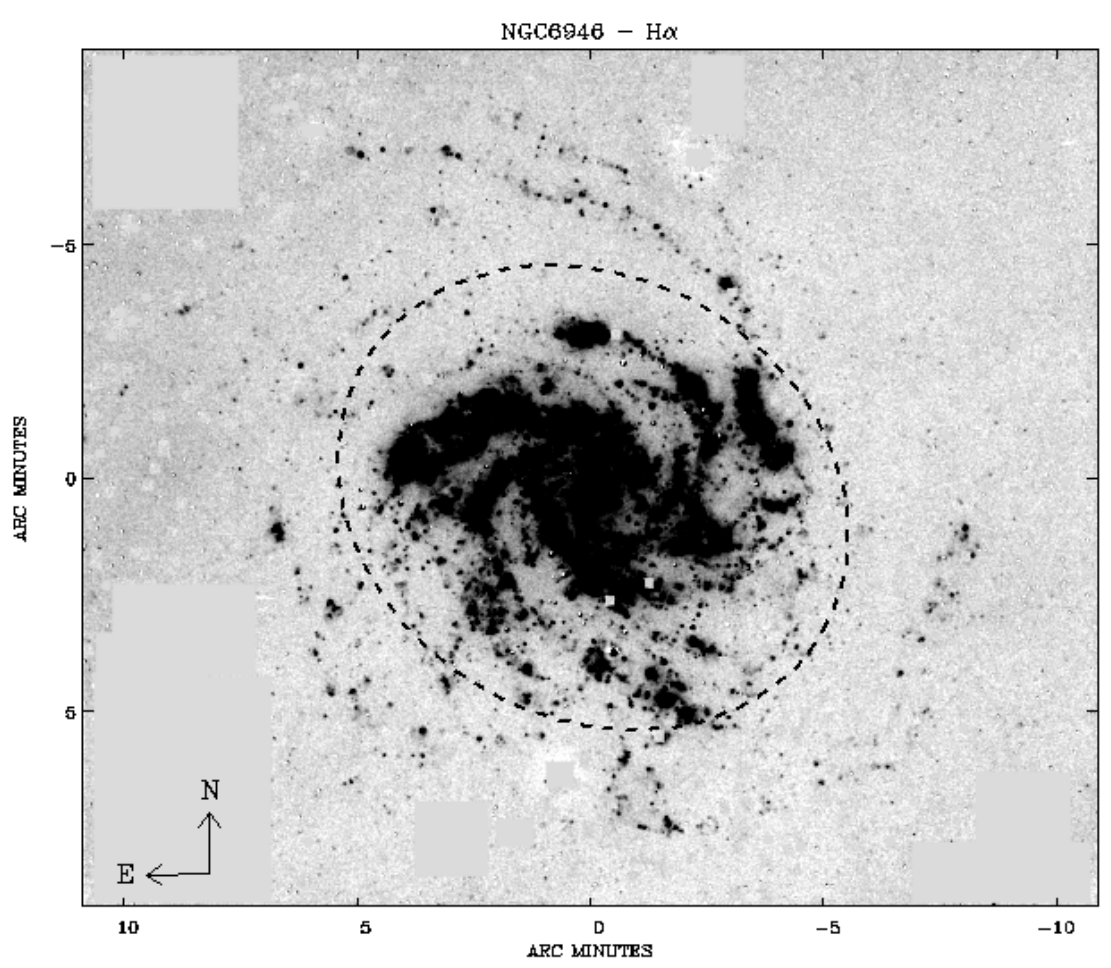

Figure 1-An $\mathrm{H} \alpha$ continuum-subtracted image of NGC 6946. The dashed line indicates $R_{25}$.

star formation diverge most in their predictions in the outer regions, and several aspects of present-day outer disks, such as gas fraction, may be similar to those of the early stages of disks in general.

\section{Highlights}

The sample consists of around 30 disk galaxies, all of which have deep $\mathrm{H} \alpha$ data, with a small sub-sample having full data: $\mathrm{H} \alpha$, broad-band and HII region spectroscopy. The study formed Annette Ferguson's PhD thesis (1997) and we shall highlight some results.

\section{1 HII Regions and Star Formation}

It is clear from the deep $\mathrm{H} \alpha$ images that many galaxies - but not all!- have HII regions, and thus very recent massive star formation, well beyond the 'optical radius' defined by the $B=25$ isophote. An immediate qualitative impression from the images (see Figure 1, NGC 6946) is that the star formation in the outer disk is exclusively in small, unclustered HII regions of typical luminosity 1-10 times that of Orion, while star formation in the inner disk $\left(R<R_{25}\right)$ occurs in HII regions that themselves cover a wide range of luminosities, and cluster into large complexes. Some of this effect is no doubt due to the simple fact that there are fewer HII regions in the outer disk, but the impression is compelling that the star formation in the outer disk is different from that in the inner disk. Why?
Fall \& Efstathiou (1980) investigated local gravitational instability in finite-thickness isothermal gas disks, deriving the criterion for Jeans' instability, giving fragmentation on scales greater than the disk thickness, $H \sim c_{\mathrm{s}}^{2} / 4 \pi G \Sigma_{\mathrm{g}}$, with $\Sigma_{\mathrm{g}}$ the surface density and $c_{\mathrm{s}}$ the sound speed in the gas. They expressed this criterion in graphical form and derived a maximum radial extent to fragmentation (their Figure 7). They predicted that gravitational fragmentation in typical disks should be confined to the inner $\sim 3$ scale-lengths, approximately equal to the optical radius, thus providing an explanation for the edges of active star formation in disk galaxies.

This result of Fall \& Efstathiou is rather underappreciated, perhaps due to its presentation in terms of their model parameters, exacerbated by the sparse observational data available at that time with which to compare their predictions. Rearranging their inequality (38) into more familiar form gives a critical gas density for gravitational instability on these scales:

$$
G \Sigma_{\mathrm{g}}>0 \cdot 3 c_{\mathrm{s}} \kappa
$$

with $\kappa$ the epicyclic frequency. The Toomre ' $Q$ ' criterion for infinitely thin, shearing gas disks yields essentially the same result for fragmentation: ${ }^{1}$

\footnotetext{
${ }^{1}$ As noted in Binney \& Tremaine (1987), section $5 \cdot 3 \cdot 1$, the similarity in instability criteria is due to the fact that the unstable scale-length in the zero-thickness case is much larger than the disk thickness.
} 

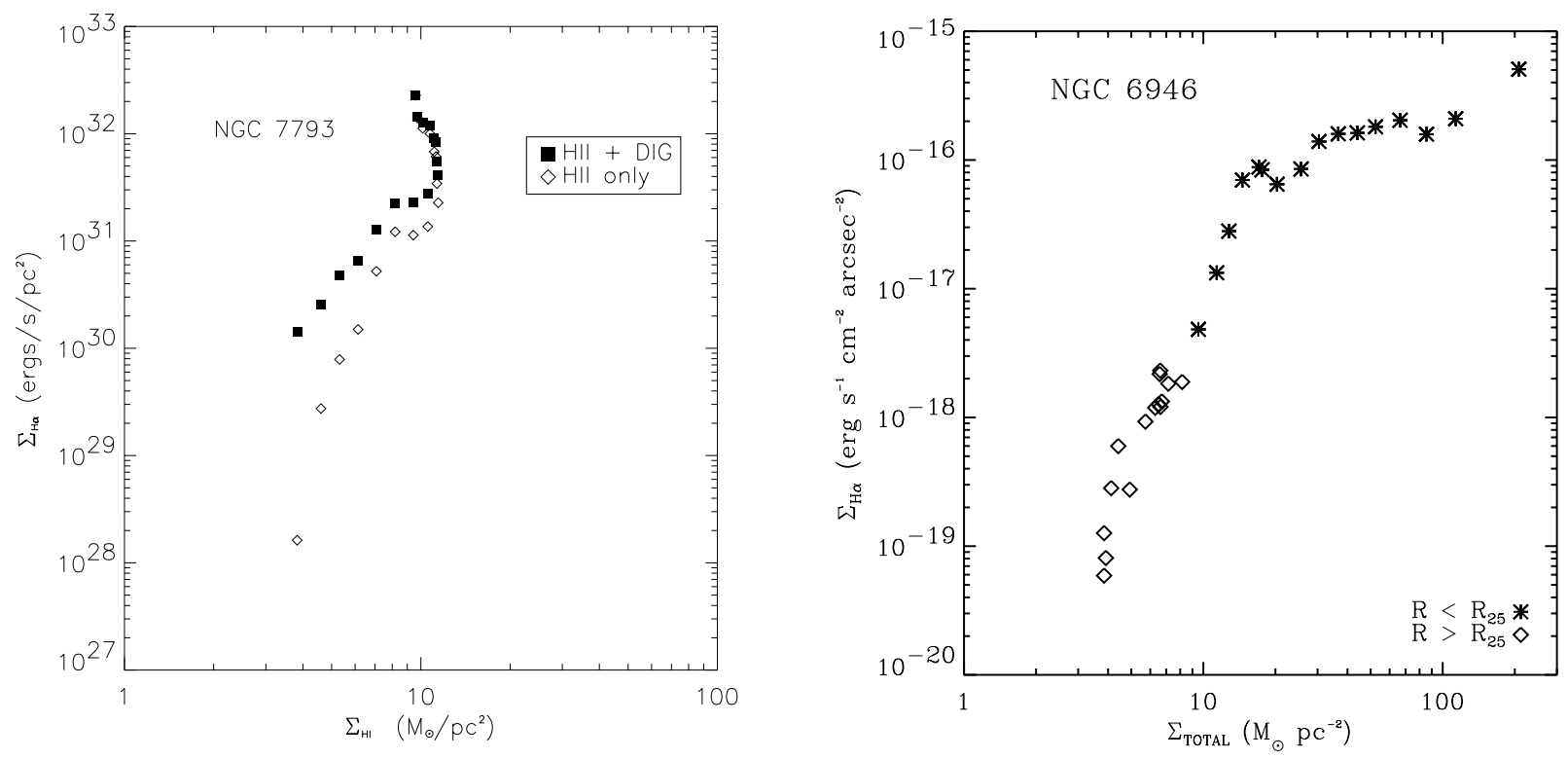

Figure 2-(Left) Variation of $\Sigma_{\mathrm{H} \alpha}$ vs $\Sigma_{\mathrm{HI}}$ for NGC 7793. Different symbols illustrate the effect that diffuse ionised gas has on the derived relationship. The 'hook' at high HI gas densities is most probably due to the neglect of molecular gas, for which data are unfortunately lacking. (Right) Variation of $\Sigma_{\mathrm{H} \alpha}$ vs $\Sigma_{\text {Gas }}$ for NGC 6946. Note the abrupt steepening of the correlation below $\Sigma_{\text {Gas }} \sim 11 M_{\odot} \mathrm{pc}^{-2}$.

$$
Q^{-1} \equiv \frac{G \Sigma_{g}}{c_{s} \kappa}>1
$$

In this case there is a maximum and minimum unstable length scale, and a fastest rate of growth at scales with wavelength $\lambda_{\text {fast }}=0 \cdot 5\left(4 \pi^{2} G \Sigma \kappa^{2}\right)$. This fastest-growing wavelength varies with radius, but is typically around $1 \mathrm{kpc}$. It is probably no coincidence that this leads to bound systems corresponding to about the scale of the largest systems in star-forming disks (Efremov 1995) and indeed of the large HII region complexes in the inner disks studied here. Of course it is not trivial to go from gravitational instability on this large scale, to star formation.

Kennicutt (1989) invoked the Toomre $Q$ criterion to explain his observed sharp drop-offs in the radial distribution of HII regions as a function of gas density. Echoing the results of Fall \& Efstathiou, Kennicutt found that the star-formation/HII region edge was typically close to the optical radius of the disk. We do see star formation beyond the optical radius, but only in small HII regions, and there is an apparent qualitative difference between the HII regions in the inner and in the outer disk. Does this change reflect crossing the $Q$-threshold? This then raises the question of what is driving the outer-disk star formation?

However, these interpretations of our data are complicated by the fact that the values of the parameters - all radially-varying - that determine the 'threshold' gas density are rather uncertain, and factors of two are important. Recent determinations of quantities such as the real radial variation of the HI gas velocity dispersion give, in some cases, edges to the gravitationally-unstable disk that are well outside even our observations. The rather dramatic change in appearance of the star-forming regions seen in our images would then be unexplained, even given the fact that $\lambda_{\text {fast }}$ varies with radius. Further, disks are not the simple one-component systems assumed in this instability analysis, and ' $Q$ ' for multi-component disks is complicated (e.g. Elmegreen 1995). It is also probably relevant that for most of the galaxies the outer-disk HII regions are in spiral arms.

Various star-formation laws may be tested with the data, and the intention is to derive the best-fitting description of all the data. A preliminary analysis shows that no one model - such as a straightforward Schmidt Law - fits all the galaxies. The variation of massive-star formation in NGC 6946 as inferred from the $\mathrm{H} \alpha$ observations is shown in Figure 2 (right panel). These data show a steepening of the 'Schmidt Law' index from inner to outer disk, as noted previously by Kennicutt (1989), but our data show this continuing with no abrupt end.

Our $\mathrm{H} \alpha$ data often allow study of diffuse gas, through surface photometry, in addition to identification and counting of HII regions. As discussed by Walterbos (1998) and by Rand (1998) (present issue pp. 99 and 106) and by ourselves (Ferguson et al. 1996a, b), the line ratios in the diffuse ionised gas, its close association with HII regions and its energy requirements are all best explained if the vast bulk of the diffuse ionised gas (DIG) is photoionised by massive stars. Inclusion of diffuse $\mathrm{H} \alpha$ emission when estimating the massive-star formation rate can 

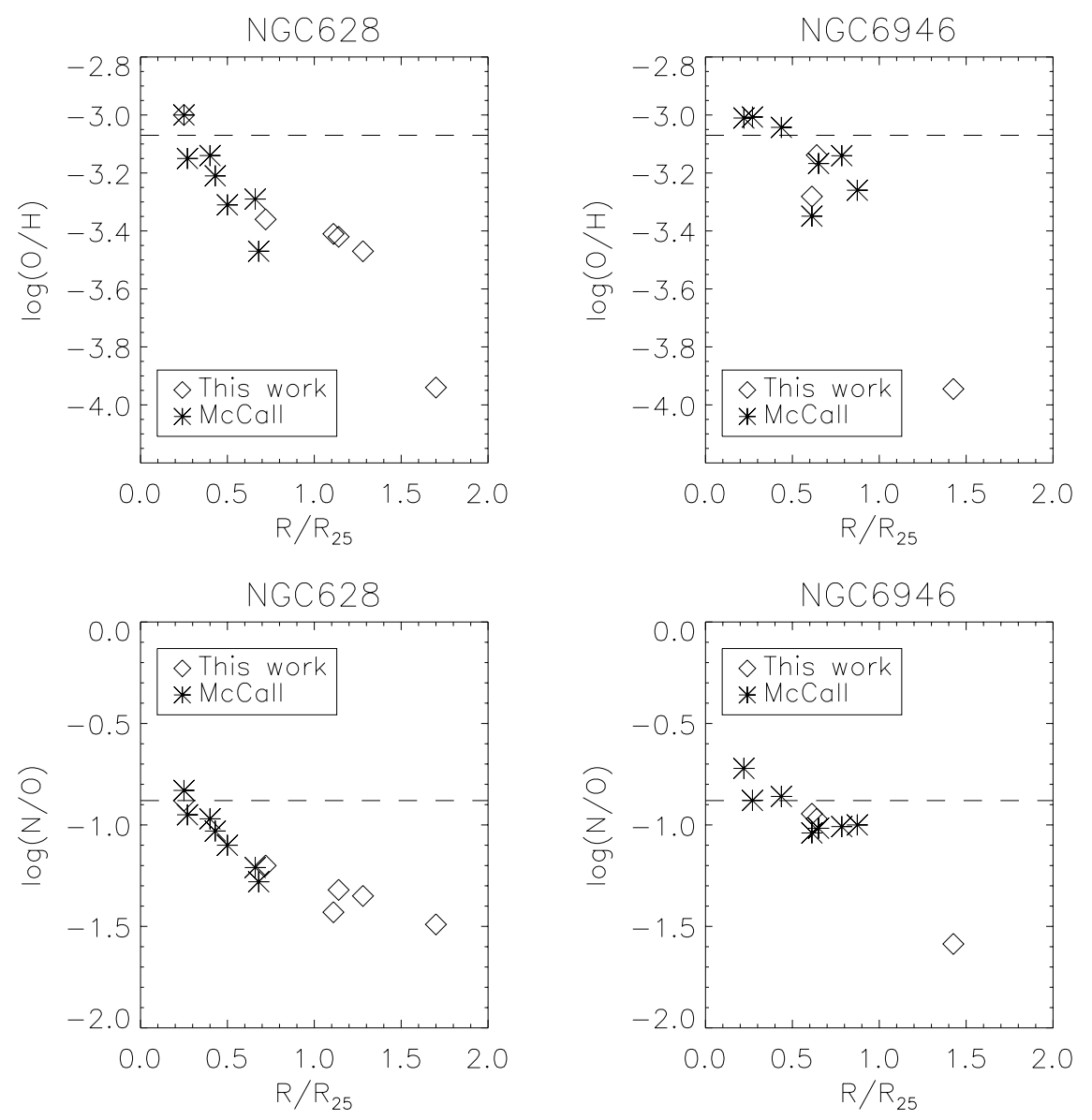

Figure 3-Chemical abundance gradients (O/H, N/O) derived for NGC 628 and NGC 6946. The dashed lines indicate the solar values.

change the derived radial profile of star formation both qualitatively and quantitatively (Ferguson et al. 1996a). Figure 2 (left panel) shows derived azimuthally-averaged star-formation rates in one of our Sd galaxies, NGC 7793, both with and without the diffuse gas, as a function of HI column density.

\subsection{Chemical Abundances in Outer Disks}

Spectroscopy of outer HII regions allows chemical abundances to be derived from the emission-line strengths. The chemical abundances are important discriminants of different theories of the temporal variation of star formation rate and gas flows, complementing the $\mathrm{H} \alpha$ data that constrain the very recent star formation rate. Again, data for the outer disk are particularly important in discriminating among theories (e.g. Prantzos \& Aubert 1995). Further, the chemical abundances of the gas-rich outer disks may be relevant for the interpretation of the metallicities seen in quasar absorption-line systems.

We have obtained the spectroscopy of several of our newly-detected HII regions in several galaxies, using the KPNO $4 \mathrm{~m}$ telescope. The galactocentric radial distances of our target HII regions extend to twice $R_{25}$. Abundances have been derived from strong lines, using the technique of McGaugh (1994) for oxygen, and that of Thurston, Edmunds \& Henry (1996) for nitrogen. A few of our HII regions in the inner disk have previous elemental abundances derived by McCall, Rybinski \& Shields (1985), and our observations and results agree well with theirs. The resultant chemical abundances are shown in Figure 3 for two galaxies; for these the outer disk is consistent with simple extrapolation of the gradient from the inner disk. Our estimates of the outer $[\mathrm{O} / \mathrm{H}]$ are typically $\sim-1$ dex, with $[\mathrm{N} / \mathrm{O}]$ from solar to one-third of solar. The nitrogen data are consistent with a secondary origin for nitrogen. These values are reminiscent of dwarf irregular galaxies (e.g. Garnett 1990), of damped Lyman- $\alpha$ clouds (e.g. Lu et al. 1996), and of low surface brightness disks (e.g. McGaugh 1994). We are developing chemical evolution models consistent with the star formation rates we infer from the $\mathrm{H} \alpha$ and broad-band observations, and augmenting our sample of HII regions with chemical abundances.

\section{Acknowledgments}

RFGW thanks Quentin Parker and all the organisers of the workshop for their help with local expenses that enabled her to attend and have such an 
enjoyable time. She also thanks all at the CfPA (UC Berkeley) for hospitality while writing this paper. JSG acknowledges support through the WFPC2 IDT.

Binney, J., \& Tremaine, S. 1987, Galactic Dynamics (Princeton University Press)

Efremov, Y. 1995, AJ, 110, 2757

Elmegreen, B. 1995, MNRAS, 275, 944

Fall, S. M., \& Efstathiou, G. P. 1980, MNRAS, 193, 189

Ferguson, A. M. N. 1997, PhD thesis, The Johns Hopkins University

Ferguson, A. M. N., Wyse, R. F. G., Gallagher, J., \& Hunter, D. A. 1996a, AJ, 111, 2265
Ferguson, A. M. N., Wyse, R. F. G., \& Gallagher, J. 1996b, AJ, 112, 2567

Garnett, D. 1990, ApJ, 363, 142

Kennicutt, R. 1989, ApJ, 344, 685

Lu, L., et al. 1996, ApJS, 107, 475

McCall, M., Rybinski, M., \& Shields, G. 1985, ApJS, 57, 1

McGaugh, S. 1994, ApJ, 426, 135

Navarro, J., \& Benz, W. 1991, ApJ, 380, 320

Navarro, J., \& Steinmetz, M. 1997, ApJ, 478, 13

Prantzos, N., \& Aubert, O. 1995, A\&A, 302, 69

Rand, R. J. 1998, PASA, 15, 106

Silk, J., \& Wyse, R. F. G. 1993, Phys. Rep. 231, 295

Thurston, T., Edmunds, M., \& Henry, R. 1996, MNRAS, 283, 990

Walterbos, R. A. M. 1998, PASA, 15, 99

Zurek, W., Quinn, P. J., \& Salmon, J. 1988, ApJ, 330, 519 